\title{
Evaluation of Alpha-fetoprotein and Prothrombin Induced by Vitamin K Absence for Hepatocellular Carcinoma Diagnosis in Patients Admitted to a Large Transplant Center
}

\author{
Razvan Cerban ${ }^{1}$, Carmen Ester ${ }^{1}$, Speranta lacob ${ }^{1}$, Mihaela Ghioca ${ }^{1}$, Liliana Paslaru ${ }^{2}$, Dumitru Radu ${ }^{3}$,
} Mugur Grasu', Georgiana Constantin ${ }^{2}$, Irinel Popescu ${ }^{4}$ and Liliana Gheorghe ${ }^{1}$

${ }^{1}$ Centre for Digestive Diseases and Liver Transplantation, Fundeni Clinical Institute, Bucharest, Romania

2Department of Biochemistry, Fundeni Clinical Institute, Bucharest, Romania

${ }^{3}$ Radiology Department, Fundeni Clinical Institute, Bucharest, Romania

${ }^{4}$ Dan Setlacec Centre of General Surgery and Liver Transplantation, Fundeni Clinical Institute, Bucharest, Romania

\section{ABSTRACT}

Background \& aims: Prothrombin induced by vitamin K absence-II (PIVKA II) is a diagnostic marker and a major prognostic factor for hepatocellular carcinoma (HCC) with limited experience in European patients. The aim of this study was to investigate the clinical utility of simultaneous measurement of alphafetoprotein (AFP) and PIVKA II for hepatocellular carcinoma (HCC) diagnosis.

Methods: In a Romanian cohort, we performed a case-control study to compare the performances of AFP and PIVKA-II serum levels for HCC diagnosis. To determine the diagnostic value of both biomarkers in distinguishing HCC from liver cirrhosis, receiver operating characteristic (ROC) curves were constructed for each biomarker and their combination.

Results: From January 2016 to December 2017, 54 cirrhotic patients and 59 HCC cases were prospectively included. ROC curve analysis showed that $63 \mathrm{mAU} / \mathrm{mL}$ and $6 \mathrm{ng} / \mathrm{mL}$ were the optimal cut-off values for PIVKA-II and AFP respectively for diagnosis of HCC in our cohort. For HCC diagnosis in patients with AFP levels $<20 \mathrm{ng} / \mathrm{mL}$, the combination of AFP and PIVKA II had a specificity of $93.8 \%$ vs. 68.8 for AFP (AUC 0.846 vs. 0.732 respectively) $(p=0.03$ ). For the diagnosis of early HCC, the combination of AFP and PIVKA-II had a sensitivity of $72.7 \%$ and a specificity of $90.9 \%$ vs. $69 \%$ and $75 \%$ for AFP (AUC 0.867 vs. 0.825 , respectively).

Conclusion: The diagnostic performance of a combination of AFP and PIVKA II is better to that of either marker alone, in the diagnosis of HCC especially in patients with AFP levels $<20 \mathrm{ng} / \mathrm{mL}$ and in patients with early tumors.

Key words: Alpha-fetoprotein, Prothrombin induced by vitamin $\mathrm{K}$ absence, Hepatocellular carcinoma

\section{INTRODUCTION}

Hepatocellular carcinoma (HCC) is one of the leading causes of cancerrelated death worldwide. It is considered to be responsible for more than
Corresponding author:

Liliana Gheorghe, MD, PhD Department of Gastroenterology and Hepatology, Fundeni Clinical Institute, Bucharest, Romania

E-mail: drgheorghe@xnet.ro

\section{Abbreviations:}

PIVKA II - Prothrombin induced by vitamin $\mathrm{K}$ absence-II HCC - hepatocellular carcinoma AFP - alphafetoprotein HCV - hepatitis C virus LC - liver cirrhosis BCLC - Barcelona Clinic Liver Cancer $\mathrm{Cl}$ - confidence intervals IQR - interquartile range

Received: 05.11.2018 Accepted: 10.12.2018

Copyright () Celsius Publishing House www.sgo-iasgo.com 
600000 new cases and 500000 deaths every year worldwide (1).

Treatment methods which can be curative, like surgical resection or liver transplantation are available only for early stage HCC patients, but most HCCs are diagnosed at an advanced stage. Therefore, early diagnosis which primarily depends on specific serum tumor markers and liver imaging tests is the key to improving the prognosis of $\mathrm{HCC}(2)$.

Since the 1970s, serum $\alpha$-fetoprotein (AFP) is the most widely-used HCC biomarker currently used by many physicians, in clinical practice for HCC diagnosis (3). The diagnostic sensitivity of AFP especially for small HCCs is rather low, with levels being elevated both in HCC patients and in $20 \%$ to $40 \%$ of those with chronic hepatitis or cirrhosis and tend to fluctuate alongside with underlying inflammatory activity (4). In patients with hepatitis $\mathrm{C}$ virus infection ( $\mathrm{HCV}$ ), which is the main cause of liver cirrhosis and the leading indication for liver transplantation in Romania, (5) AFP is often elevated in the absence of HCC, but has o normal value in as many as $50 \%$ of patients with $\mathrm{HCC}(6)$.

The current Western guidelines $(7,8)$ have excluded the measurement of AFP for HCC diagnosis, because of its limited value in detecting HCC especially during the early disease stages, with a sensitivity of around $60 \%$ at a cut-off value of $20 \mathrm{ng} / \mathrm{mL}(8,9,10)$ and low specificity (11).

PIVKA II is an abnormal form of prothrombin protein, with no coagulation function, that is present at high levels in the serum of HCC patients (12).

Since the initial report by Liebman et al (13), PIVKA II has been considered to be a highly specific biomarker for diagnosis as well as a predictor of prognosis in patients with $\operatorname{HCC}(9,14,15)$.

Several studies demonstrated that measurement of PIVKA II in conjunction with AFP has a sensitivity ranging from $47.5 \%$ to $94.0 \%$, and a specificity ranging from $53.3 \%$ to $98.5 \%$ for early HCC diagnosis, and that the combination is superior to either marker used alone $(16,17)$. Pote et al. also showed PIVKA II utility for the diagnosis of early HCC and demonstrated that it can predict microvascular invasion (18).

For these reasons Japanese guidelines on HCC management recommended the use of of the combination of AFP and PIVKA II for routine follow up of patients with a high risk of HCC development (19). The cut-offs of AFP $>200 \mathrm{ng} / \mathrm{mL}$ and DCP $>40 \mathrm{mAU} / \mathrm{mL}$ are considered by the same Japanese guidelines to be highly sugestive for HCC diagnosis.

Despite other reports $(20,21,22)$ also demonstrating that a combined analysis of AFP and PIVKA II led to better detection of HCC, there are still some controversies regarding the diagnostic accuracy of PIVKA II and AFP combination.

Many studies mostly from Asia have compared the utility of tumor markers for HCC diagnosis, but a headto-head comparison of the diagnostic utility of AFP, PIVKA II and their combination in a Eastern European cohort has not been reported yet.

The aim of this study was to evaluate the diagnostic performance of AFP, PIVKA II and their combination among patients with HCC admitted to a transplant center.

\section{MATERIALS AND METHODS}

\section{Patients}

We conducted a case control study at Fundeni Clinical Institute from January 2016 to December 2017.

A total of 59 patients with $\mathrm{HCC}$ and 54 controls with liver cirrhosis (LC) were enrolled. All HCC patients were newly diagnosed. Plasma samples were obtained from all the patients included in the study regardless if they belonged to the case of the control group. HCC was diagnosed based on histology or typical image characteristics as defined by the EASL and AASLD guidelines $(7,8)$ Barcelona Clinic Liver Cancer (BCLC) system was used for HCC staging (8).

LC was diagnosed by one or more findings of portal hypertension including: cirrhotic appearance of the liver with splenomegaly on imaging studies, thrombocytopenia (platelet $<110,000 / \mathrm{mm}^{3}$ ), finding esophageal varices on endoscopy, detection of ascites, or presence of hepatic encephalopathy. All LC patients underwent imaging studies to exclude HCC.

Clinical and laboratory data were prospectively colected for all subjects and included liver transaminases, bilirubin, albumin, creatinine, platelet count, INR hepatitis B and C serologies.

For this study, liver disease etiology was matched as much as possible between the HCC and the LC groups.

This study was conducted with approval from the Ethics Committee of the Fundeni Clinical Institute. An informed consent in written was obtained from all included participants.

\section{Sample storage and assays}

Two peripheral blood samples were collected in EDTA tubes from each patient at diagnosis for the HCC group or at the time of the visit in our clinic for the control group. Aliquot samples were stored at $-80^{\circ} \mathrm{C}$ 
until measurement.

Serum AFP and PIVKA-II levels were measured for each sample by the same experienced laboratory technician, who was blind to all clinical data. For the frozen samples that were thawed we did not perform further refreezing.

Serum AFP and PIVKA II were determined by use of automated chemiluminescent microparticle immunoassays (ARCHITECT AFP 3P36; ARCHITECT PIVKA-II 2P4) according to the manufacturer's instructions, utilizing the Abbott $^{\mathrm{TM}}$ Architect iSystem 1000 analyser in the Department of Biochemistry at Fundeni Clinical Institute, Bucharest. For each ELISA plate used, an adequate standard curve was calculated.

\section{Statistical analysis}

All enrolled patients were included in the final analysis. Continuous variables were presented as means + SD (standard deviation) or as median when suitable. Categorical variable were reported as percentages.

For evaluating the differences between patients in the HCC group and patients in the LC group we used a chi-square test for classified variables and MannWhitney test was performed for continuous nonparametric variables. Significance was considered for $\mathrm{p}<0.05$.

For the evaluation of the diagnostic performance, receiver operating characteristics (ROC) curves and binary logistic regression analyses were constructed for each biomarker and for their combination. The optimal cut-off value was considered by determining the Youden index for each ROC curve using a single marker. To identify the relationships between the biomarkers a correlation analysis was performed. For distinguishing between $\mathrm{HCC}$ and LC patients, the differences between the area under the ROC curve (AUC) for each biomarker and their $95 \%$ confidence intervals $(\mathrm{Cl})$ were calculated. Evaluation of the correlation of selected variables was performed using Pearson's correlation coefficient.

Statistical analyses were performed using Medcalc ver. 13.3.3.0 software.

\section{RESULTS}

Both the clinical and biological characteristics of the study population $(n=103)$ are presented in table 1 . The HCC patient group had a higher proportion of males $(p=0.04)$ and was older $(p=0.03)$ than the LC control group. All HCC patients had underlying liver cirrhosis. $\mathrm{HCV}$ infection was the most com $\neg$ mon etiology in both groups, followed by hepatitis B infection. There were no significant differences according to Child-Pugh class between the two patient groups.

Because the serum levels of biomarkers were sometimes extremely high, we measured median and interquartile range (IQR) values to allow more accurate comparisons and avoid the extreme outliers that can

Table 1 - Clinical characteristics of the study population

\begin{tabular}{|c|c|c|c|}
\hline & HCC $(n=59)$ & LC $(n=54)$ & $\mathrm{p}$ value \\
\hline Age, median years (range) & $60.3 \pm 8,52$ & $59.4 \pm 8.4$ & $p=0.03$ \\
\hline Gender, $\mathrm{n}$ (male) & $39(66.1 \%)$ & $28(51.9 \%)$ & $p=0.04$ \\
\hline \multicolumn{4}{|l|}{ Etiology } \\
\hline $\mathrm{HCV}$ & $37(62.7 \%)$ & $31(57.4 \%)$ & $p=0.86$ \\
\hline HBV & $14(23.7 \%)$ & $13(24.1 \%)$ & \\
\hline Alcohol & $7(11.9 \%)$ & $8(14.8 \%)$ & \\
\hline Others & $1(1.7 \%)$ & $2(3.7 \%)$ & \\
\hline \multicolumn{4}{|l|}{ Child-Pugh class } \\
\hline$A$ & $46(77.9 \%)$ & $31(57.4 \%)$ & $p=0.24$ \\
\hline B & $10(16.9 \%)$ & $13(24.1 \%)$ & \\
\hline $\mathrm{C}$ & $3(5.1 \%)$ & $10(18.5 \%)$ & \\
\hline Platelet, $\times 10^{3} / \mathrm{L}$ & $99(74.50-139.50)$ & $81(67.0-133.0)$ & $p=0.36$ \\
\hline Albumin, $g / L$ & $3.7(3.08-4.0)$ & $3(2.7-3.5)$ & $p=0.01$ \\
\hline Bilirubin, mg/dL & $1.8( \pm 1.4)$ & $4.8( \pm 3.7)$ & $p<0.0001$ \\
\hline $\mathrm{ALT}, \mathrm{UI} / \mathrm{L}$ & $54(37-109)$ & $49(32-115)$ & $p=0.65$ \\
\hline INR & $1.3( \pm 0.3)$ & $1.6( \pm 0.5)$ & $p=0.006$ \\
\hline MELD score & $10.4( \pm 3.2)$ & $14.1( \pm 5.2)$ & $\mathrm{p}<0.0001$ \\
\hline AFP, $n g / m L$ & $18(6.63-104.56)$ & $4.75(3.21-6.70)$ & $p<0.0001$ \\
\hline PIVKA II, mUA/mL & $188.4(81.36-723.21)$ & $63(34.73-160.03)$ & $p<0.0001$ \\
\hline
\end{tabular}


produce misleading mean values. Median serum AFP and PIVKA-II levels were found to be significantly higher in patients with HCC than in the LC group (AFP, 18 (IQR 6.63-104.56) vs. 4.75 (IQR (3.21-6.70) ng/mL; PIVKA- II 188.4 (IQR 81.36-723.21), vs 63 (IQR 34.73-160.03) $\mathrm{mUA} / \mathrm{mL}$. There was no correlation between the serum levels of AFP and PIVKA II ( $r=0.107)$ (fig. 1).

Staging of $\mathrm{HCC}$ was done using BCLC system. BCLC staging was 0 in $16.9 \%, A$ in $38.9 \%, B$ in $20.3 \%, C$ in $18.6 \%$ and $D$ in $5.1 \%$ of the HCC patients. Subgrup analysis of cases showed that patients with BCLC C and D vs. $0, A, B$ had a significant higher serum, PIVKA II levels (779.6 vs. $160.5 \mathrm{mAU} / \mathrm{mL}, \mathrm{p}=0.01$ ) (fig. 2). For AFP there was no statistically significant difference between patients with $\mathrm{BCLC}$ grade of $\mathrm{C}$ and $\mathrm{D}$ vs. $0, \mathrm{~A}$ and $\mathrm{B}$ $(p=0.78)$.

\section{Biomarkers performance evaluation}

The performance of AFP, PIVKA II and their combination for diferenciating HCC from LC was evaluated on all pacients included in study and on two subgroups of HCC cases. A comparison of AUC, sensitivity, and specificity for differentiating HCC from $L C$ in a subgroup of HCC patientrs with AFP level $<20 \mathrm{ng} / \mathrm{mL}$ and in another subgroup of patients with early stage $\mathrm{HCC}$ were performed. Early stage HCC included BCLC stage 0 and A patients.

Performance assessment on all patients included in the study showed that the combination of AFP + PIVKA II had the best AUC $(0.862,95 \% \mathrm{Cl} 0.774$ 0.926) for HCC diagnosis, compared with the AUC for AFP (cut-off: $6 \mathrm{ng} / \mathrm{mL}$ ) alone of 0.831 (95\% Cl 0.734 0.899 ) and for PIVKA-II (cut-off $63 \mathrm{mAU} / \mathrm{mL}$ ), 0.759 (95\% Cl $0.658-0.842$ ) (fig. 3).

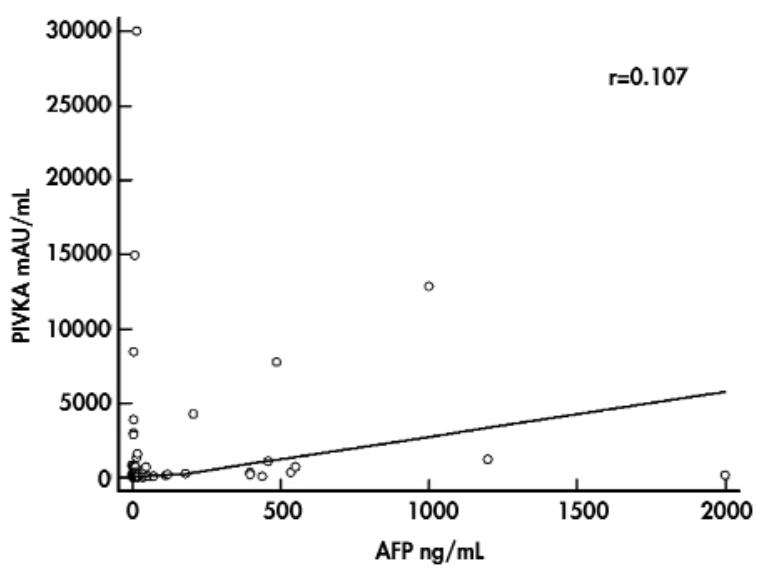

Figure 1 - Linear correlation between AFP and PIVKA II
At the cut-off value of $63 \mathrm{mAU} / \mathrm{mL}$ PIVKA II was proved to be more sensitive ( $81.4 \%$ ) but less specific than AFP (table 2).

In our cohort, 36 (61\%) HCC and 46 (85.2\%) LC patients had a plasma AFP $<20 \mathrm{ng} / \mathrm{mL}$. As a single marker PIVKA II $>63 \mathrm{mAU} / \mathrm{mL}$ showed the best AUC $(0.751,95 \% \mathrm{Cl} 0.621-0.840)$ (fig. 4) with a sensitivity of $81.4 \%$ and a specificity of $62.5 \%$ (table 2). The combination of both biomarkers, (AFP $>6 \mathrm{ng} / \mathrm{mL}$ and PIVKA-II > $63 \mathrm{mAUmL}$ ) showed an AUC of 0.862 which is statisticaly significant better than for AFP alone $(p=0.03)$.

In the present study, 33 (55.9\%) HCC patients were diagnosed at an early stage (BCLC 0 or A). AFP as a single marker for the diagnosis of early $\mathrm{HCC}$, had the best (AUC $0.82595 \% \mathrm{Cl} 0.712-0.908$ ) with a sensitivity of $69.7 \%$ and a specificity of $75.7 \%$ (AFP $>6 \mathrm{ng} / \mathrm{mL}$ ). AFP was most specific as a single marker (75.7\%). Although PIVKA-II had a greater sensitivity than AFP for early HCC diagnosis, the specificity was lower (60.6\%) (PIVKA $>63 \mathrm{mAU} / \mathrm{mL}$ ) than that of AFP. The combination of both biomarkers, (AFP > 6ng/mL and PIVKA-II $>63 \mathrm{mAUmL}$ ) showed an AUC of 0.867 which is better than each marker alone but the difference was not statistically significant (fig. 5) (table 2).

\section{DISCUSSION}

Early HCC diagnosis is of great importance to ensure that curative interventions can be applied to improve the prognosis and survival of patients (23). In addition to ultrasound imaging and other methods, clinicians greatly depend on serum markers, such as AFP, for HCC diagnosis, especially in areas where advanced diagnostic technologies are not readily available.

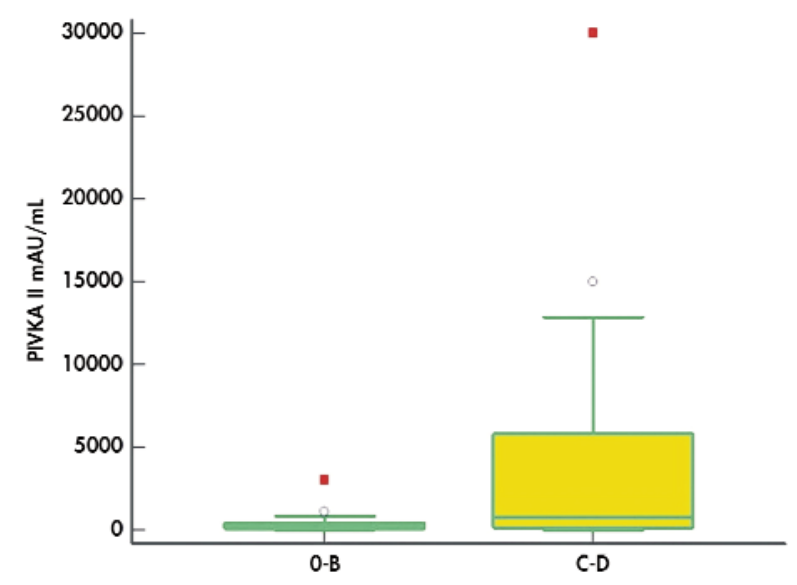

Figure 2 - Box plot of PIVKA II levels according to BCLC stage 


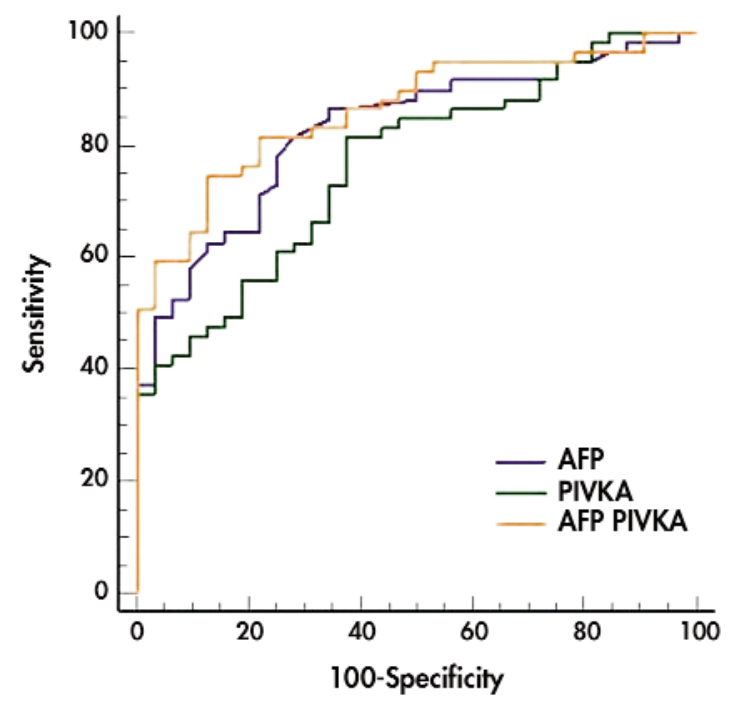

\begin{tabular}{|c|c|c|c|c|c|}
\hline HCC marker & AUC & $95 \% \mathrm{Cl}$ & \multicolumn{2}{|c|}{$P$ (Delong test) } \\
${$\cline { 1 - 3 }$} }$ & 0.831 & $0.734-0.899$ & \multirow{2}{*}{$\mathrm{P}=0.32$} & \\
\cline { 1 - 3 } $\mathrm{PIVKA} \mathrm{II}$ & 0.759 & $0.658-0.842$ & & \multirow{2}{*}{$\mathrm{P}=0.04$} & \\
\hline AFP + PIVKA II & 0.862 & $0.774-0.926$ & & \\
\hline
\end{tabular}

Figure 3 - ROC curve for all HCC cases

Although AFP is used by physicians for assessing HCC besides imaging techniques, it is not a completely reliable marker and it is not recommended by current guidelines due to low sensitivity and specificity. This makes AFP insufficient for early diagnosis with subsequent loss of the possibility for an effective HCC treatment.

The aim of our study was to determine whether a combination of AFP and PIVKA II could be useful for
HCC di-agnosis compared to the traditional AFP for the first time in Romanian patients.

Since HCC development is one of the most frequent causes of death in LC patients (24) and many patients with $\mathrm{HCC}$ have underlying $\mathrm{LC}$, we took the decision to include only $\mathrm{LC}$ patients as a control group.

In the current study HCC patients were older and more often males than patients who did not have HCC. Male predominance in HCC development may be

Table 2 - Diagnostic value of AFP, PIVKA-II, and their combination in discriminating HCC from LC in different study groups

\begin{tabular}{|c|c|c|c|c|c|}
\hline HCC marker & Sn & Sp & PPV & NPV & AUC \\
\hline \multicolumn{6}{|l|}{ HCC vs. LC - all patients } \\
\hline $\mathrm{AFP}>6 \mathrm{ng} / \mathrm{mL}$ & $78.0 \%$ & $75.8 \%$ & $85.2 \%$ & $65.8 \%$ & 0.831 \\
\hline PIVKA II >63mAU/mL & $81.4 \%$ & $60.6 \%$ & $78.7 \%$ & $64.5 \%$ & 0.759 \\
\hline AFP + PIVKA II & $74.6 \%$ & $87.5 \%$ & $91.7 \%$ & $65.1 \%$ & 0.862 \\
\hline \multicolumn{6}{|c|}{ HCC vs. LC - patients with AFP $<20 \mathrm{ng} / \mathrm{mL}$} \\
\hline $\mathrm{AFP}>6 \mathrm{ng} / \mathrm{mL}$ & $75.1 \%$ & $68.8 \%$ & $73.0 \%$ & $64.1 \%$ & 0.732 \\
\hline PIVKA II >63mAU/mL & $80.6 \%$ & $62.5 \%$ & $70.0 \%$ & $72.4 \%$ & 0.751 \\
\hline AFP + PIVKA II & $66.7 \%$ & $93.8 \%$ & $88.9 \%$ & $72.5 \%$ & 0.846 \\
\hline \multicolumn{6}{|c|}{ HCC vs. LC - early stage HCC patients } \\
\hline AFP $>6 n g / m L$ & $69.7 \%$ & $75.7 \%$ & $74.2 \%$ & $71.4 \%$ & 0.825 \\
\hline PIVKA $\|>63 \mathrm{mAU} / \mathrm{mL}$ & $76.7 \%$ & $60.6 \%$ & $65.8 \%$ & $72.6 \%$ & 0.710 \\
\hline AFP + PIVKA II & $72.7 \%$ & $90.9 \%$ & $88.9 \%$ & $78.9 \%$ & 0.867 \\
\hline
\end{tabular}

$\mathrm{HCC}=$ hepatocellular carcinoma, $\mathrm{LC}=$ liver cirrhosis, PIVKA $\|=$ Prothrombin induced by vitamin $\mathrm{K}$ absence $\mathrm{II}$,

$A F P=$ alpha-fetoprotein, $S n=$ sensitivity, $S p=$ specificity, $P P V=$ positive predictive value, $N P V=$ negative predictive value, $A \cup C=$ area under the curve 


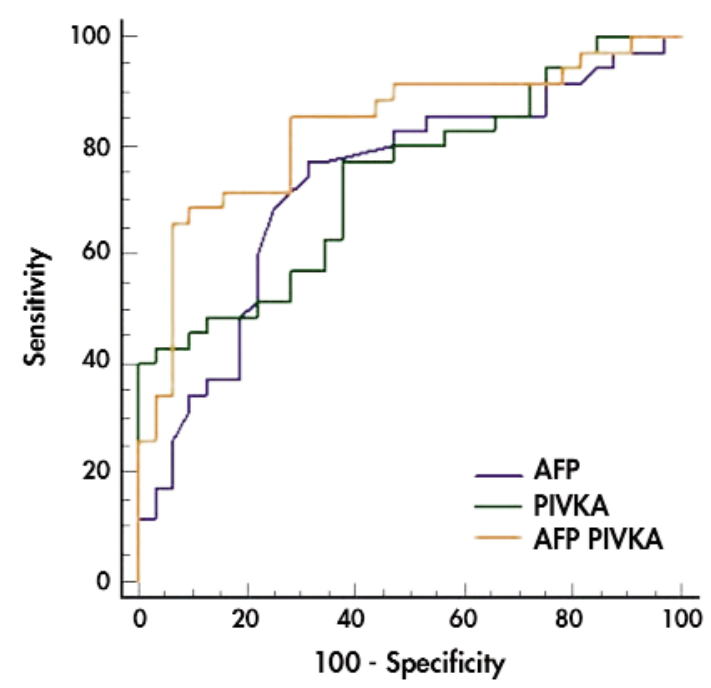

\begin{tabular}{|c|c|c|c|c|c|}
\hline HCC marker & AUC & $95 \% \mathrm{Cl}$ & \multicolumn{2}{|c|}{$P$ (Delong test) } \\
\hline AFP & 0.732 & 0.618 to 0.837 & \multirow{2}{*}{$P=0.93$} & & \multirow{2}{*}{$\mathrm{P}=0.03$} \\
\cline { 1 - 3 } PIVKA II & 0.751 & 0.621 to 0.840 & & \\
\hline AFP + PIVKA II & 0.846 & 0.726 to 0.915 & & \\
\hline
\end{tabular}

Figure 4 - ROC curve for HCC cases with low AFP

explained by hepatocyte androgenic stimulation and by greater exposure to toxins (25).

Chronic hepatitis $C$ is thought to be the main cause of cirrhosis and HCC in Western countries (26). In our study, chronic HCV hepatitis was the most common cause in both $\mathrm{HCC}$ and $\mathrm{LC}$ control group. The results from our study are therefore likely to be representative of the total Romanian population.

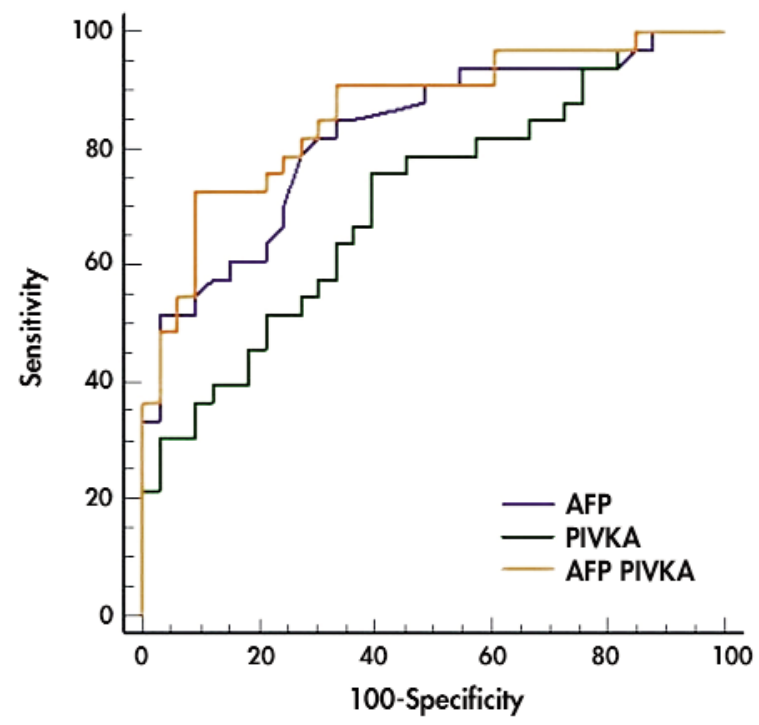

\begin{tabular}{|c|c|c|c|c|c|}
\hline HCC marker & AUC & $95 \% \mathrm{Cl}$ & \multicolumn{2}{|c|}{$\mathrm{P}$ (Delong test) } \\
\hline AFP & 0.825 & $0.712-0.908$ & \multirow{2}{*}{$\mathrm{P}=0.15$} & & \multirow{2}{*}{$\mathrm{P}=0.37$} \\
\cline { 1 - 3 } $\mathrm{PIVKA} \mathrm{II}$ & 0.710 & $0.585-0.815$ & & \\
\hline AFP + PIVKA II & 0.867 & $0.755-0.935$ & & \\
\hline
\end{tabular}

Figure 5 - ROC curve for early stage HCC 
In the present study we demonstrated that increased serum concentration of both AFP and PIVKA Il were significantly corelated with the diagnosis of HCC. This finding is similar to other authors who reported that PIVKA II produced by HCC cells is an invasive and proangiogenic factor that stimulates tumoral growth $(14,27)$.

In accordance to other previous papers, in our study group there was no correlation between the serum levels of PIVKA II and AFP which let us to evaluate the diagnostic performance of their combination.

The serum concentration of PIVKA II increased at higher BCLC staging, but AFP levels had no correlation with tumor stage. This finding might be explained by the fact that, as previously reported, tumor diameter is significantly correlated with PIVKA II levels (28).

The cut-off values used for each biomarker were fixed based on the value that resulted in the largest AUC. Although in each subgroup analysis the cut-offs were slightly different, to avoid confusion from changing the cut-offs for different clinical scenarios we took the decision to use the same cut-off value for each biomarker for easier use in real-world practice.

For HCC diagnosis, the optimal cut-off value of PIVKA-II, which resulted after ROC curve analysis was $63 \mathrm{mAU} / \mathrm{mL}$. According to previous studies, the optimal cut-off value for PIVKA-II as a diagnostic biomarker for $\mathrm{HCC}$, has been estimated to range from 30 to 42 $\mathrm{mAU} / \mathrm{mL}$ and its variability may be due to the preexisting liver disease $(18,22)$.

The cut-off value of PIVKA-II for HCC diagnosis in the present study is higher than previous papers. An explanation might be the fact that since serum PIVKA-II levels in patients with HCC will gradually increase with liver disease progression, the inclusion of more advanced patients as opposed to other studies may account for the different optimal diagnostic cut-off value in our study as compared to previous papers.

We were able to demonstrate that PIVKA-II (cut-off: $63 \mathrm{mAU} / \mathrm{mL}$ ) had a higher sensitivity but a lower specificity that AFP in all the study groups. The combination of AFP and PIVKA II at the above cut-off increased overall specificity and positive predicted value at the expense of sensitivity.

In the subgroup of patients with AFP $<20 \mathrm{ng} / \mathrm{mL}$, PIVKA II showed the best sensitivity (80.6\%) and the best AUC $(0.751,95 \% \mathrm{Cl})$, as a single marker. Accuracy of PIVKA II was slightly superior to AFP in this subgroup of patients but without statistical significance.

Previous studies have shown that PIVKA-II could be helpful for the early diagnosis of small HCC tumors $(29,30)$.
The combination of AFP $>6 \mathrm{ng} / \mathrm{mL}$ and PIVKA II $>63 \mathrm{mAU} / \mathrm{mL}$ had the highest AUC in the early HCC patients subgroup $(0.867,95 \% \mathrm{Cl})$, with a sensitivity of $72.7 \%$ and a specificity of $90.9 \%$ (table 2 ). For early HCC diagnosis PIVKA II had a sensibility of $76.7 \%$ vs. $69 \%$ for AFP, but the specificity was lower, $60.6 \%$ vs $75.7 \%$ for AFP. These findings are consistent with previous studies which demonstrated that for this group of patients PIVKA II had a better sensibility but worse specificity than AFP (9).

Our results are slightly different from a French paper [18] in which the authors demonstrated that for early HCC patients, PIVKA II had a better diagnostic performance that AFP, with a sensitivity of $77 \%$ (vs. $75.7 \%$ ) and specificity of $82 \%$ (vs. $61 \%$ ), at a cut-offs values of $42 \mathrm{mAU} / \mathrm{mL}$ for PIVKA II and $5.5 \mathrm{ng} / \mathrm{mL}$ for AFP.

PIVKA II testing is convenient, inexpensive, and might be a valuable addition to current options for the HCC diagnosis, but clinical decision should be made taking into account that various factors might influence the diagnostic performance of this biomarker. Since levels of both AFP and PIVKA II are higher in HCC patients than in non-HCC patients and healthy individuals, different cut-off levels can be tried to further improve their specificity.

In our study both biomarkers and their combination were demonstrated to have a moderate accuracy for HCC diagnosis. The present study demonstrated, by performing a head-to-head comparison of the two diagnostic markers of HCC, that AFP was the best single marker and the combination of AFP with PIVKA-II has the best diagnostic performance, in all study groups, compared to either marker alone.

This study was subject to several limitations. One limitation was that the samples were obtained from our center only, and therefore our results require external validation. Repeated experiments for internal validation were carried out strictly. Refreezing of thawed plasma was avoided.

Another limitation of this study is that the study population was small, and we used only one commercially available ELISA PIVKA II assay. The enrolled patients with HCC were heterogeneous in regard to underlying liver disease etiology, status and tumor stage which reflects real-world practice, but this fact may also lead to variability. Finally, the aim of the present study was to compare the clinical utility of AFP and PIVKA II for HCC diagnosis with easy-to-use cut-off values, not to evaluate their performance for surveillance. 


\section{CONCLUSION}

In conclusion, PIVKA II as a single marker does not appear to be significantly superior to AFP for HCC diagnosis in our cohort of patients. The combination of AFP and PIVKA II may serve as a valuable addition to current imaging modalities for HCC diagnosis, because it greatly improves the diagnostic performance for HCC especially in patients with low AFP levels and in patients with early stage tumors.

Future studies performed on larger cohorts are needed to confirm our results, with the need to establish the most efficient and economical current biomarker combination.

\section{Conflict of interest}

All author declare that they have no conflict of interest.

\section{REFERENCES}

1. Ferlay J, Shin HR, Bray F, Forman D, Mathers C, Parkin DM. GLOBOCAN 2008 v2.0, Cancer Incidence and Mortality Worldwide: IARC Cancer Base No. 10. Lyon, France: International Agency for Research on Cancer; 2010.

2. Tamura Y, Igarashi M, Suda T, Wakai T, Shirai Y, Umemura T. et al. Fucosylated fraction of alphafetoprotein as a predictor of prognosis in patients with hepatocellular carcinoma after curative treatment. Dig Dis Sci. 2010;55:2095-2101.

3. Chung MY, Choi MS, Paik SW, Ahn BH, Lee JH, Koh KC, et al. Early diagnosis and improved survival with screening for hepatocellular carcinoma. Clin Mol Hepatol. 2003:9(2):116-125.

4. Johnson PJ. The role of serum alpha-fetoprotein estimation in the diagnosis and management of hepatocellular carcinoma. Clin Liver Dis. 2001:5:145-59.

5. Gheorghe L, Sporea I, lacob S, Sirli R, Trifan A, Dobru D. et al. Position paper on treatment of hepatitis C in Romania, 2017. Part one. J Gastrointestin Liver Dis. 2017;26:171-181

6. Ertle JM, Heider D, Wichert M, Keller B, Kueper R, Hilgard P, et al. Combination of $\alpha$-Fetoprotein and Des-\&\#947;-Carboxy Prothrombin Is Superior in Detection of Hepatocellular Carcinoma. Digestion 2013; 87:121-131

7. Bruix J, Sherman M, American Association for the Study of Liver Disease. Management of hepatocellular carcinoma: an update. Hepatology. 2011;53(3):1020-1022.

8. European Association for the Study of the Liver, European Organisation for Research Treatment of Cancer. EASL-EORTC clinical practice guidelines: management of hepatocellular carcinoma. J Hepatol 2012; 56(4):908-943.

9. Marrero JA, Feng Z, Wang Y, Nguyen MH, Befeler AS, Roberts LR, et al. Alpha-fetoprotein, desgamma carboxyprothrombin, and lectin-bound alpha-fetoprotein in early hepatocellular carcinoma. Gastroenterology. 2009;137(1):110-118.

10. Adachi Y, Tsuchihashi J, Shiraishi N, Yasuda K, Etoh T, Kitano S AFP-producing gastric carcinoma: multivariate analysis of prognostic factors in 270 patients. Oncology 2003; 65(2):95-101.

11. Chaiteerakij R, Addissie BD, Roberts LR. Update on biomarkers of hepatocellular carcinoma. Clin Gastroenterol Hepatol 2015;13(2): 237-245.

12. Carr BI, Kanke F, Wise M, Satomura S. Clinical evaluation of lens culinaris agglutinin-reactive alpha-fetoprotein and des-gammacarboxy prothrombin in histologically proven hepatocellular carcinoma in the United States. Dig Dis Sci. 2007;52:776-782;

13. Liebman HA, Furie BC, Tong MJ, Blanchard RA, Lo KJ, Lee SD. et al. Des-gamma-carboxy (abnormal) prothrombin as a serum marker of primary hepatocellular carcinoma. N Engl J Med 1984; 310:1427-31

14. Koike $\mathrm{Y}$, Shiratori $\mathrm{Y}$, Sato S, Obi S, Teratani T, Imamura M. et al. Des-gamma-carboxy prothrombin as a useful predisposing factor for the development of portal venous invasion in patients with hepatocellular carcinoma: a prospective analysis of 227 patients. Cancer 2001;91:561-9.

15. Imamura H, Matsuyama $\mathrm{Y}$, Miyagawa $\mathrm{Y}$, Ishida $\mathrm{K}$, Shimada $\mathrm{R}$, Miyagawa $S$. et al. Prognostic significance of anatomical resection and des-gamma-carboxy prothrombin in patients with hepatocellular carcinoma. Br J Surg 1999;86:1032-8.

16. Shimauchi $Y$, Tanaka M, Kuromatsu $R$, Ogata $R$, Tateishi $Y$, Itano $S$. et al. A simultaneous monitoring of Lens culinaris agglutinin A-reactive alpha-fetoprotein and desgamma-carboxy prothrombin as an early diagnosis of hepatocellular carcinoma in the follow-up of cirrhotic patients. Oncol Rep 2000;7:249-56.

17. Durazo FA, Blatt LM, Corey WG, Lin JH, Han S, Saab S. et al. Des-gamma-carboxyprothrombin, alpha-fetoprotein and AFP-L3 in patients with chronic hepatitis, cirrhosis and hepatocellular carcinoma. J Gastroenterol Hepatol 2008;23:1541-8.

18. Pote N, Cauchy F, Albuquerque M, Voitot H, Belghiti J, Castera L, et al. Performance of PIVKA-II for early hepatocellular carcinoma diagnosis and prediction of microvascular invasion. J Hepatol 2015; 62:848-54.

19. Omata M, Cheng A-L, Kokudo N, Kudo M, Lee JM, Jia J. et al. AsiaPacific clinical practice guidelines on the management of hepatocellular carcinoma: a 2017 update. Hepatology International. 2017; 11(4):317-370

20. Sultanik P, Ginguay A, Vandame J, Popovici T, Meritet JF, Cynober L. et al. Diagnostic accuracy of des-gamma-carboxy prothrombin for hepatocellular carcinoma in a French cohort using the Lumipulse® G600 analyzer. J Viral Hepat. 2017;24(1):80-85.

21. Caviglia GP, Abate ML, Petrini E, Gaia S, Rizzetto M, Smedile A. Highly sensitive alpha-fetoprotein, Lens culinaris agglutinin-reactive fraction of alpha-fetoprotein and des-gamma-carboxyprothrombin for hepatocellular carcinoma detection. Hepatology Research. 2016; 46:E130-E5.

22. Ji J, Wang H, Li Y, Zheng L, Yin Y, Zou Z. et al. Diagnostic Evaluation of Des- Gamma-Carboxy Prothrombin versus alpha-Fetoprotein for Hepatitis B Virus-Related Hepatocellular Carcinoma in China: A Large-Scale, Multicentre Study. Plos One. 2016; 11.

23. Llovet JM, Burroughs A, Bruix J. Hepatocellular carcinoma. Lancet 2003:362:1907-17.

24. El-Serag HB, Davila JA, Petersen NJ, McGlynn KA. The continuing increase in the incidence of hepatocellular carcinoma in the United States: an update. Ann Intern Med 2003;139:817-23.

25. Lui WY, Lin HL, Chau GY, Liu TY, Chi CW. Male predominance in hepatocellular carcinoma: New insight and a possible therapeutic alternative. Med Hypotheses. 2000;55(4):348-50.

26. Kim MN, Kim BK, Han KH. Hepatocellular carcinoma in patients with chronic hepatitis $\mathrm{C}$ virus infection in the Asia-Pacific region. $\mathrm{J}$ Gastroenterol 2013:48:681-8.

27. Fujikawa T, Shiraha H, Yamamoto K. Significance of des-gammacarboxy prothrombin production in hepatocellular carcinoma. Acta Med Okayama. 2009;63(6):299-304.

28. Cerban R, Ester C, lacob S, Paslaru L, Dumitru R, Grasu M, et al. Evaluation of tumor response using alpha-fetoprotein and des-gamma-carboxy prothrombin in hepatocellular carcinoma patients who underwent transarterial chemoembolization. Chirurgia (Bucur). 2018:113(4):524-533.

29. Saitoh S, Ikeda K, Koida I, Tsubota A, Arase Y, Chayama K, et al. Serum des-gamma-carboxyprothrombin concentration determined by the avidin-biotin complex method in small hepatocellular carcinomas. Cancer 1994;74:2918-23.

30. Mita Y, Aoyagi Y, Yanagi M, Suda T, Suzuki Y, Asakura H. et al. The usefulness of determining desgamma- carboxy prothrombin by sensitive enzyme immunoassay in the early diagnosis of patients with hepatocellular carcinoma. Cancer 1998;82:1643-8 